\title{
World Journal of Recommendations for control and Pediatric Surgery prevention of infections for pediatric orthopedics during the epidemic period of COVID-19
}

Chunxing Wu (D) , Zhiqiang Zhang, Yueqiang Mo, Dahui Wang (D) , Bo Ning, Ping Xu, Jun Song, Xinhong Pei, Xiaoyan Ni, Yiming Zheng, Tian Xia, Yanhui Jing, Wanlin Sun, Junrong Meng, Chuang Qian

To cite: Wu C, Zhang Z, Mo Y, et al. Recommendations for control and prevention of infections for pediatric orthopedics during the epidemic period of COVID-19. World Jnl Ped Surgery 2020;3:e000124. doi:10.1136/wjps-2020-000124

$\mathrm{CW}, \mathrm{ZZ}$ and YM contributed equally.

$\mathrm{CW}, \mathrm{ZZ}$ and YM are joint first authors.

Received 5 March 2020

Revised 17 March 2020

Accepted 17 March 2020
Check for updates

(C) Author(s) (or their employer(s)) 2020. Re-use permitted under CC BY-NC. No commercial re-use. See rights and permissions. Published by BMJ.

Pediatric Orthopedics, Naitonal Children's Medical Center, Children's Hospital of Fudan University, Shanghai, China

Correspondence to Dr Dahui Wang; wangdahui@ fudan.edu.cn

Dr Bo Ning; ningb022776@ aliyun.com

\section{ABSTRACT}

The outbreak of Coronavirus Disease 2019 (COVID-19) caused by severe acute respiratory syndrome coronavirus 2 (SARS-CoV-2) emerged and spread rapidly throughout the world. As of February 29, 2020, 79389 cases of COVID-19 have been reported, and the outbreak is linked to 2838 deaths. The population is generally susceptible to the disease, and differences in incubation periods after infection exist among individuals. These two aspects of COVID-19 pose significant challenges to pediatric orthopedic diagnosis and treatment. As a dedicated center for managing pediatric cases of SARS-CoV-2 in Shanghai, our hospital has mobilized all branches and departments to undertake joint actions for scientific prevention and control, precise countermeasure and comprehensive antiepidemic efforts. Combined with our experience, we have consulted the relevant national regulations and the latest research advances and have formulated the prevention and control measures of SARS-CoV-2 infection, including outpatient, emergency, inpatient and surgical cares, for clinical practices of pediatric orthopedics according to the physicochemical properties of SARS-CoV-2. It may serve as practical references and recommendations for managing SARS-CoV-2 infection in other pediatric specialties and in other hospitals.

Severe acute respiratory syndrome coronavirus 2 (SARS-CoV-2) ${ }^{1}{ }^{2}$ caused pneumonia Coronavirus Disease 2019 (COVID-19) began to ravage and spread worldwide. SARS-CoV-2 infection has been classified as category B infectious disease legally but is managed as a category A infectious disease. Our hospital is the only one in Shanghai designated for the admission of SARS-CoV-2-infected children. The department of infectious diseases has admitted dozens of suspected and confirmed cases of children. ${ }^{3} 4$ During the epidemic period, especially after the Spring Festival, millions of people returned to work. Pediatric orthopedic outpatient, emergency and operations will be inevitable. SARS-CoV-2

\section{Summary box}

What is already known about this subject?

- Severe acute respiratory syndrome coronavirus 2 (SARS-CoV-2) caused pneumonia COVID-19 began to ravage and spread throughout the world Physicochemical properties of SARS-CoV-2, clinical features and diagnosis and treatment program of COVID-19 have been promulgated by the National Health Commission of China. The most important goal is to control infectious sources. Protection of medical staff is obviously a crucial component of epidemic prevention and control.

What are the new findings?

- In our hospital, a recommendation working group "Recommendation Formulating Team for Pediatric Orthopedic Infection controls during the Epidemic's Period of COVID-19" was formed, to focus on relevant issues for protection of medical staff in pediatric orthopedics other departments. Combined with our experience, we have consulted the relevant national regulations and the latest research advances and have formulated the prevention and control measures of SARS-CoV-2 infection, including outpatient, emergency, inpatient and surgical cares for pediatric orthopedics, pediatric surgery and others. We have formulated "Requirements for protection supplies in different grade (table 1)" and "Control and surgical handling flows of pediatric orthopedics at outpatient and emergency clinics in responses to COVID-19 infection (figure 1)".

How might it impact on clinical practice in the foreseeable future?

- These recommendations, which are formulated to protect medical staff in pediatric orthopedics and patients, are also expected to serve as a reference and recommendations for other pediatric specialties and/or other hospitals.

is transmitted between humans mainly via aerial droplets and direct contact. ${ }^{56}$ Thus far, no proven and specific antiviral treatments 
for COVID-19 are available. ${ }^{7}$ Therefore, the most important goal is to control infectious sources. Protection of medical staff is obviously a crucial component of epidemic prevention and control. By consulting relevant national regulations, the latest research progress and guidance from the advisory group, our department developed these recommendations, which were also expected to serve as a reference and recommendations for other pediatric specialties and/or other hospitals.

\section{APPLIED POPULATION}

Medical staff of pediatric orthopedic outpatient and emergency department, hospital administrator and staff of auxiliary department.

\section{APPLICABLE PERSONNEL}

Pediatric orthopedic patients and accompanying personnel.

\section{RECOMMENDATION FORMATION PROCESS}

Given the high demand of patients for medical treatment and the need to protect medical staff from infectious diseases, a recommendation working group "Recommendation Formulating Team for Pediatric Orthopedic Infection controls during the Epidemic's Period of COVID-19" (including all authors) was formed to focus on relevant issues for protection of medical staff in pediatric surgery, pediatric orthopedics, infectious diseases department, anesthesiology department, and nursing department to hospital administrators.

1. Building and refining the first level catalog: applied population, applicable personnel, general principles, physicochemical properties of SARS-CoV-2 and clinical features of COVID-19. Prevention and control procedures in outpatient clinic, emergency department, inpatient and surgical cares of pediatric orthopedics. "Ordinary patient", "risky patient" and "suspected patient" protection.

2. Establishing the second level catalog and retrieving the current evidence of COVID-19 for the first and second level directory problem.

3. Writing draft proposals.

4. Synthesizing suggestions at all levels to form discussion papers.

5. Discussing the proposal face to face in the proposal working group to form the applicable draft.

6. Trying out the recommendations, gathering feedback and forming a formal version of the recommendations.

\section{GENERAL PRINCIPLES}

COVID-19 has a general susceptibility in the population. There were many reports of nosocomial infection in literature and media, including medical staffs and hospitalized patients. Therefore, $\mathrm{WHO}^{8}$ has published a recommendation for both patients and doctors, based on current knowledge of the situation in China and other countries. The strategies to prevent or limit transmission in healthcare settings include the following.

1. Ensuring triage, early recognition, and source control (isolating patients with suspected SARS-CoV-2 infection).

2. Applying standard precautions for all patients.

3. Implementing empiric additional precautions (droplet and contact and, whenever applicable, airborne precautions) for suspected cases of SARS-CoV-2 infection.

4. Implementing administrative controls.

5. Using environmental and engineering controls.

Based on that, in order to reduce the iatrogenic infection and exposure infection, combined with our experience, we developed a set of recommendations for the department of pediatric surgeries, orthopedics and other relevant department. Patients, families and medical staffs should adhere to the following recommendations.

1. Control the number of accompanying family members.

2. Evaluate the epidemiological history and condition of the children and their close contacts in the first 14 days before the visit.

3. Hospitalization and surgical indications should be strictly controlled; selective operation is not allowed.

4. Pay attention to the protection of medical staff during perioperative period.

5. Decrease hospitalization days.

6. Set up the transition ward, one room for one patient and continuous evaluation. Do the best to avoid SARSCoV-2 spread and cross infection.

\section{PHYSICOCHEMICAL PROPERTIES OF SARS-COV-2 AND CLINICAL FEATURES OF COVID-19}

SARS-CoV-2 is the seventh coronavirus that has been found to infect humans. It is a $\beta$ category coronavirus, in the same category as the coronavirus that caused Middle East respiratory syndrome (MERS) and severe acute respiratory syndrome (SARS). Spike glycoprotein (S) on virus surface is the main antigen of coronavirus and can be used for typing. The nuclear protein of the virus can be used for antigen diagnosis. Most understanding of the physical and chemical properties of SARS-CoV-2 comes from studies on SARS-CoV and MERS-CoV. The virus is sensitive to ultraviolet and heat, which can be inactivated by heating at $56^{\circ} \mathrm{C}$ for 30 minutes and by using lipid solvents such as $75 \%$ ethanol, chlorine-containing disinfectant, peroxyacetic acid and chloroform, but not by chlorhexidine. ${ }^{9}$

The transmission characteristics of COVID-19 are as follows.

1. Infection sources. The main sources of the infection are patients infected by COVID-19 with or without clinical symptoms. The incubation period is between 1 and 14 days, with a median incubation period of 3 days and a maximum of 24 days. ${ }^{10}$

2. Transmission route. The novel virus is spread through respiratory droplets when patients cough, talk loudly 
or sneeze. Close contact is also a source of transmission. Aerosol and fecal-oral transmission of digestive tract are also possible, while it needs to be further clarified.

3. Susceptible population. The age range of the reported infected population was 36 hours old to 96 years old, with no significant gender difference. The elderly and people with basic diseases are more likely to become severe cases. Children may have mild clinical symptoms after infection. ${ }^{11}$ Nevertheless, medical personnel still need to pay more attention, closely monitor, and strive for early identification and timely treatment.

"Diagnosis and Treatment Program of 2019 New Coronavirus Pneumonia (trial sixth version)" released by the National Health Commission of China on February 18, 2020 has defined as follow.

\section{Suspected cases}

1. Epidemiological history. (1) Travel or residence history in Wuhan, other areas with recent local transmission of COVID-19, or the local community with confirmed patient. (2) Close contact with patient with laboratory-confirmed COVID-19 (positive for nucleic acid test). (3) Close contact with people from Wuhan or surrounding areas or local communities with fever or respiratory symptoms case report. (4) Children who are related with a cluster outbreak or close contact with COVID-19-infected cases.

2. Clinical manifestation. (1) Fever and/or respiratory symptoms. (2) Imaging features of viral pneumonia. In the early stage of pneumonia cases, chest images show multiple small patchy shadows and interstitial changes, remarkable in the lung periphery. Severe cases can further develop to bilateral multiple ground-glass opacity, infiltrating shadows and pulmonary consolidation, with infrequent pleural effusion. (3) Normal or low white blood cell count or reduced lymphocyte in early onset. Patients should be suspected of COVID-19 infection if they meet any one of the criteria in the epidemiological history and any two of the criteria in clinical manifestations. If there is no clear history of epidemiology but the three clinical manifestations are met, the case is deemed to be suspected too.

\section{Confirmed cases}

Suspected cases who meet any one of the following criteria.

1. Respiratory tract or blood samples tested positive for COVID-19 nucleic acid using reverse transcription polymerase chain reaction (RT-PCR).

2. Genetic sequencing of respiratory tract or blood samples is highly homologous with the known COVID-19.

On this basis, our hospital also issued clinical practice guidelines for children. ${ }^{4}$ Screening of children at risk was enhanced through epidemiological history and clinical manifestations.

Although the clinical manifestations of the confirmed infection in children are mild, the clinical manifestations vary. Among the confirmed cases of children with clinical data collected, fever and cough are more common, as well as fatigue, myalgia, nasal congestion, runny nose, sneezing, pharyngeal pain, headache, dizziness, nausea, vomiting, abdominal pain and diarrhea. ${ }^{9}$ This means that some patients with coronavirus infection are not first diagnosed with respiratory symptoms such as cough, fever and chest pain. A small number of patients will have muscle pain, abdominal pain and diarrhea as the first main complaint; pediatric orthopedic surgeons and pediatric surgeons should pay attention to these conditions during the outbreak, pay attention to screening for COVID-19 and take measures to prevent the spread of SARS-CoV-2 infection in the hospital.

\section{CONTROL AND SURGICAL HANDLING FLOW OF PEDIATRIC ORTHOPEDICS AT OUTPATIENT AND EMERGENCY CLINICS IN RESPONSES TO SARS-COV-2 INFECTION}

According to the characteristics of SARS-CoV-2 infection, national and hospital requirements for prevention and control, ${ }^{49}$ combined with clinical practice in pediatric orthopedics, we specially formulated the outpatient, emergency, inpatient and surgical procedures for prevention and control of SARS-CoV-2 infection in pediatric orthopedics (figure 1).

Nurses in outpatient or emergency service will measure patients' temperature and ask epidemiological history, fever, respiratory symptoms at first, fill the epidemiological survey, and make a record about children and their accompanying guardians' identity card information, containing contact number, address and possible close contact with SARS-CoV-2 carriers after they passing the incubation period. Pediatric orthopedist in the outpatient and emergency department has to check and confirm the patient's epidemiological survey again, collect the survey and turn back to preexamination nurses for centralized preservation.

We divided the children into the following three categories.

1. "Ordinary patient": without history of epidemiology or related symptoms.

2. "Risky patient": a history of epidemiology without related symptoms.

3. "Suspected patient": with a history of epidemiology and/or related symptoms, the patient should be transferred to the "fever clinic" in the isolation building for screening and diagnosis.

For children with different categories, we clarify different levels of protection and specific requirements for different conditions (see table 1 ). ${ }^{4}$

\section{THE PROTECTION OF "ORDINARY PATIENT'”"}

1. It is recommended that if the children are not in the emergency department, they should not seek medical treatment at the hospital to prevent the occurrence of cross-infection due to dense staff. At the peak of the epidemic, selective surgery was temporarily suspended, 


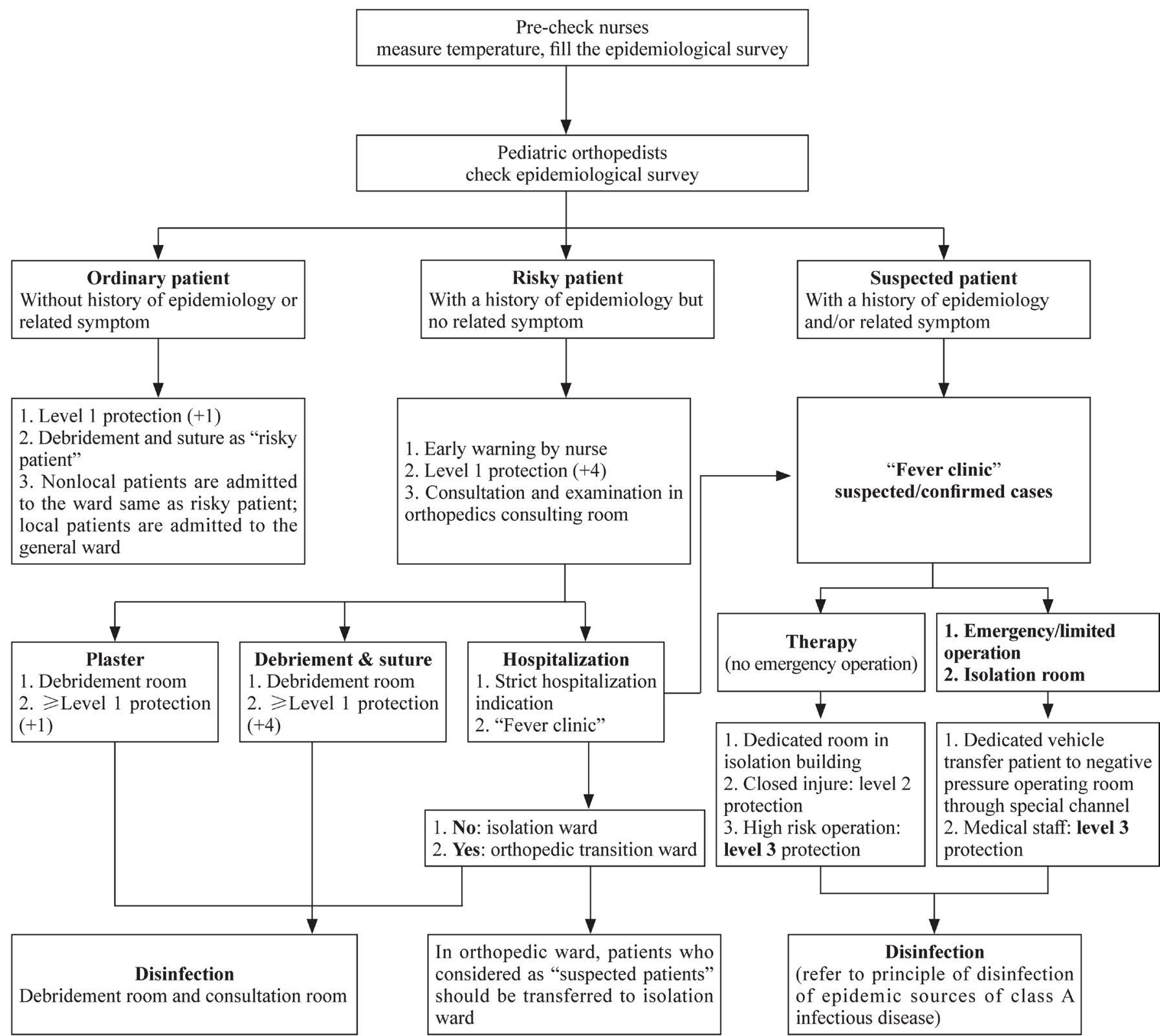

Figure 1 Control and surgical handling flows of pediatric orthopedics at outpatient and emergency clinics in responses to SARS-CoV-2 infection.

and then resumed gradually after the epidemic stabilized. With good precautions, pediatric orthopedists only carry out emergency operation and limited operation. If hospitalization is required, patients will be admitted to the general ward.

2. General protection for outpatient and emergency orthopedists: at least level 1 protection $(+1)$ : wearing medical overalls, medical surgical masks, medical caps and latex gloves.

3. Ask each patient and attendant to wear masks. This cannot only prevent cross-infection between patients, but also effectively protect medical staffs.

4. Improve the medical environment, set up waiting areas, maintain medical order and prevent crossinfection of patients.
5. The consultation area should be well ventilated and regularly cleaned and disinfected.

6. During the epidemic, hand hygiene should be strictly implemented, in accordance with the WHO five hand hygiene moments, following the "seven-step hand washing method".

7. Protection for high-risk operations, such as debridement and suture, is the same as "risky patients" (see below). If hospitalization is needed, the hospitalization process for patient from other cities is the same as for "risky patients" (see below). Patient needs to stay in the transition ward at first (for single person and single room admission management, the location of the transition ward should be avoided as far as possible from the entrance of the ward passage. The transition 
Table 1 Requirements for protection supplies in different grades

\begin{tabular}{|c|c|c|c|c|c|c|c|c|}
\hline & & el & prot & tion & & & & \\
\hline & 0 & 1 & +1 & +2 & +3 & +4 & Level 2 protection & Level 3 protection \\
\hline Medical overalls & $\sqrt{ }$ & $\sqrt{ }$ & $\sqrt{ }$ & $\sqrt{ }$ & $\sqrt{ }$ & $\sqrt{ }$ & $\sqrt{ }$ & $\sqrt{ }$ \\
\hline Medical mask & $\sqrt{ }$ & & & & & & & \\
\hline Medical surgical mask & & $\sqrt{ }$ & $\sqrt{ }$ & $\sqrt{ }$ & $\sqrt{ }$ & & & $\sqrt{ }$ \\
\hline Medical protective mask & & & & & & $\sqrt{ }$ & $\sqrt{ }$ & $\sqrt{ }$ \\
\hline Medical cap & $\sqrt{ }$ & $\sqrt{ }$ & $\sqrt{ }$ & $\sqrt{ }$ & $\sqrt{ }$ & $\sqrt{ }$ & $\sqrt{ }$ & $\sqrt{ }$ \\
\hline Goggles/face shield & & & & & $\sqrt{ }$ & $\sqrt{ }$ & $\sqrt{ }$ & \\
\hline Protective face shield & & & & & & & & $\sqrt{ }$ \\
\hline Medical isolation gown & & & & $\sqrt{ }$ & $\sqrt{ }$ & $\sqrt{ }$ & & \\
\hline Medical protective clothing & & & & & & & $\sqrt{ }$ & $\sqrt{ }$ \\
\hline Shoe covers & & & & & & & $\sqrt{ }$ & $\sqrt{ }$ \\
\hline Latex gloves & & & $\sqrt{ }$ & $\sqrt{ }$ & $\sqrt{ }$ & $\sqrt{ }$ & $\sqrt{ }$ & $\sqrt{ }$ \\
\hline
\end{tabular}

Protective face shield: full protective breathing face shield/full protective electric air supply filter respiratory.

ward should have a clear warning sign), and can be transferred to a general ward after three days of isolation and observation. Local patients are admitted to the general ward according to the normal procedures during non-epidemic situations. If there are sufficient beds in the transition ward, they are recommended to stay in the transition ward at first. "Recommended Admissions and Management of Infection Prevention and Control of Children with Pediatrics During COVID-19 Epidemic Period (1st edition)"12 is warranted for the inpatients. If patients are suspected as "suspected patients" during hospitalization, he/she will be transferred to isolation wards.

8. Note that "ordinary patients" may stay in the SARSCoV-2 infection incubation, so close follow-up is also necessary.

\section{THE PROTECTION OF "RISKY PATIENT"}

For patients with epidemic history of SARS-CoV-2 but no symptoms, we take the following preventive measures (the carriers are in the incubation period but still at risk of spreading the virus).

1. The orthopedist, both in outpatient and emergency department, will be informed of the situation of the patients by nurses, as the pre-warning.

2. The orthopedist shall adopt at least level 1 protection $(+4)$ : wearing medical overalls, medical caps, medical protective masks, goggles, medical isolation gowns and double latex gloves.

3. Inquiring clinical history and performing physical examination merely in outpatient or emergency department with full protection.

4. Patients who need auxiliary examination should wear medical surgical masks, accompanied by special personnel of the hospital. Relevant auxiliary departments should be informed in advance to take preventive measures, and return to the orthopedic clinic in time after the examination.

5. After the treatment, the surfaces in the clinic are wiped with $1000 \mathrm{mg} / \mathrm{L}$ chlorine-containing disinfectant for 10 minutes. Then, ultraviolet radiation is used to sterilize the air for 40 minutes. Based on the results of physical and radiological examination, the orthopedic surgeon shall make a relevant decision following numbers principles.

(1) If the condition is mild, let the patient leave the hospital after treatment in the orthopedic clinic.

(2) Closed fractures needing a plaster immobilization should be performed in debridement department. The orthopedist should be defended at least level 1 protection $(+1)$ : wearing medical overalls, medical surgical masks, medical caps and latex gloves.

(3) If the patient needs to observe the change of illness, the patient should not be left in the arbitrary observation room. The SARS-CoV-2 infection should be excluded by "clinic fever" physicians before transferring to the orthopedic transition ward. Try to provide single rooms for isolated children, and reduce the chance of contact with the co-residents. After hospitalization, please refer to "Diagnosis, treatment, and prevention of 2019 novel coronavirus infection in children: experts' consensus statement". ${ }^{12}$

(4) If the patient needs to be hospitalized for surgery, ask physician to rule out the possibility of a suspected infection. If the infection is suspected or confirmed, follow the "suspected child" protection procedure (see below). If COVID-19 infection is excluded, such patients will be admitted to the orthopedic transition ward with separate treatment management.

(5) If the patient needs to undergo debridement and suture operations without hospitalization, the following debridement and suture procedures should be followed: orthopedist and other personnel should have at 
least level 1 protection $(+4)$ in a separate debridement room: medical overalls, medical caps, medical protective masks, face masks/goggles, medical isolation gowns and bilateral latex gloves. If tetanus vaccination is needed, human immunoglobulin (non-skin test type) should be selected and injected in the debridement room by the injector who has the same protection with orthopedist. Dedusting garbage treatment: the garbage is placed in an airtight bucket with two layers of yellow garbage bags. The garbage is sprayed with $5000 \mathrm{mg} / \mathrm{L}$ chlorine-containing disinfectant and the bag is tightly sealed. After debridement, debridement room should be disinfected: the object surface is wiped and disinfected with $1000 \mathrm{mg} / \mathrm{L}$ chlorinecontaining disinfectant, acting on for $>10$ minutes. The air was sterilized by ultraviolet irradiation for 40 minutes. ${ }^{4}$

\section{THE PROTECTION OF "SUSPECTED PATIENT" (INCLUDING CONFIRMED PATIENTS)}

1. If the patient is considered to be a suspected case, the patient should first be transferred to the 'fever clinic' for screening of SARS-CoV-2 infection. If confirmed as a suspected case, the patient should be isolated immediately and reported to the hospital. Nucleic acid tests are also performed twice to determine the presence of SARS-CoV-2 infection.

2. The following orthopedic surgeons will bring their own equipment and wear protective equipment in the infectious disease isolation building according to the prevention and control requirements. Generally, level 2 protection is provided for closed injuries and level 3 protection is provided for high-risk operations (table 1$)$.

3. After experts' discussion, it is clear that if emergency or limited operation is needed, the patient will be transferred to the negative pressure operating room through the special channel by special transport vehicle. Special attention should be paid to the isolation and the following protection during the perioperative period of suspected or confirmed children. (1) Reduce surgical participants. (2) The operation should avoid rush hour and personnel flow. (3) Prevent cross infection in the operating room. For example, disposable equipment should be used as much as possible in the operating room. Equipment unrelated to the operation should be moved out of the operating room, and those that cannot be moved out should be covered with protective film. During the operation, surgeons, nurses, anesthesiologists and other relevant personnel need take at least level 3 protection: wearing double medical caps, medical protective masks (inner) and medical surgical masks (outer), protective face shield, medical protective clothing (inner) and medical isolation gowns (outer), double latex gloves and shoe covers. After the operation, the surgeons take off the medial isolation gowns (outer), the outer medical surgical mask, the outer medical cap and the outer latex gloves in the operating room. After hand disinfection by hydrogen peroxide, the surgeons take off the medical protective clothing (inner), remove the inner latex gloves, then carry out hand disinfection again with the disinfectant containing hydrogen peroxide. The operating room shall be sterilized according to the principle of disinfection of epidemic sources of class A infectious disease. ${ }^{4}$ The object surface of the operating room is wiped and disinfected with $2000 \mathrm{mg} / \mathrm{L}$ chlorine-containing disinfectant, acting on for $>10$ minutes. The air is atomized with hydrogen peroxide or hypochlorite for onehour. Medical waste and medical fabric waste bags containing $5000 \mathrm{mg} / \mathrm{L}$ chlorine-containing preparations are sprayed and disinfected and treated as infectious medical waste and fabric. Postoperative medical waste is placed in an airtight bucket with two layers of yellow garbage bags, and is sprayed with $5000 \mathrm{mg} / \mathrm{L}$ chlorine-containing disinfectant to sterilize it.

Occupational exposure during surgery, such as surgical instrument damage to protective gloves or protective clothing, can cause contact with body fluids to medical personnel. If this occurs, stop the operation immediately, remove the protection according to the above-mentioned principles, then deal with it according to the "Emergency Treatment Procedure After Sharp Instrument Injury" issued by the Infection Quality Control Center. Report the incidence to the relevant departments of the hospital and fill in the "occupational exposure registration form". The relevant departments will conduct further isolation or clear diagnosis.

All medical personnel involved in the operation need to be isolated for observation after the operation. If the suspected patient is excluded from SARS-CoV-2 infection, the isolation should be lifted. If the patient is diagnosed with the SARS-CoV-2 infection, all medical personnel involved in the operation are quarantined for 14 days. When presenting with suspicious symptoms, he/she should seek medical treatment in time. During the isolation period, medical staff communicated with other staff in the department through mobile phones to avoid contact with colleagues who were not involved in contact with such patient.

\section{CONCLUSION}

In conclusion, those are the clinical protective measures summarized in pediatric orthopedics, hoping to provide reference for pediatric orthopedics and other related departments. For suspected cases, we need to provide timely contact to professional doctors for judgment and treatment. At the same time, according to the changes of national epidemic prevention and control measures, to optimize protection process and measures are needed. In view of the changing epidemic situation, when there is a conflict between the treatment 
of pediatric orthopedic diseases and the prevention and control of the epidemic situation, it is necessary to make overall consideration and focus on the prevention and control of the epidemic situation.

Acknowledgements Recommendation Formulating Team for Pediatric Orthopedic Infection controls during the Epidemic's Period of COVID-19: Kuiran Dong and Shan Zheng (pediatric surgery); Gongbao Liu (medical department); Ruixue Ma, Dahui Wang, Bo Ning, Chunxing Wu, Hong Chen, Jun Song, Yiming Zheng, Yueqiang Mo, Zhiqiang Zhang,Ping Xu, Xinhong Pei, Xiaoyan Ni, Tian Xia, Yanhui Jing, Wanlin Sun, Junrong Meng, Chuang Qian and Chunhui Cai (pediatric orthopedics); Xuan Wang and Zhijian Zhou (department of anesthesiology); Guoping Lu (critical care medicine); Chuanqing Wang (nosocomial infection control department); Mei Zeng (infectious diseases division); Zhongwei Qiao (radiology department); Peihong Xu (operating room); Jin Xu (clinical laboratory division); Guomei Shen (outpatient and emergency department)

Contributors WCX, ZZQ and MYQ contributed equally to this article. They contributed to the design, reporting, describing and interpretation of the study. WDH and NB were responsible for the overall content as guarantors. They planed, proposed and executed the study. XP, SJ, PXH, NXY, ZYM, XT,JYH, SWL, MJR and QC drafted part of the manuscript and approved the final version.

Funding The authors have not declared a specific grant for this research from any funding agency in the public, commercial or not-for-profit sectors.

Competing interests None declared.

Patient consent for publication Not required.

Ethics approval Not required for this review article.

Data availability statement All data relevant to the study are included in the article or uploaded as supplementary information. All text and data relevant to this study are included in this article, data are available in public and will be published in public.

Open access This is an open access article distributed in accordance with the Creative Commons Attribution Non Commercial (CC BY-NC 4.0) license, which permits others to distribute, remix, adapt, build upon this work noncommercially, and license their derivative works on different terms, provided the original work is properly cited, appropriate credit is given, any changes made indicated, and the use is non-commercial. See: http://creativecommons.org/ licenses/by-nc/4.0/.
ORCID iDs

Chunxing Wu http://orcid.org/0000-0002-2702-5000

Dahui Wang http://orcid.org/0000-0002-3101-702X

\section{REFERENCES}

1 Zhu N, Zhang D, Wang W, et al. China novel coronavirus investigating and research team. A novel coronavirus from patients with pneumonia in China, 2019. N Engl J Med 2020.

2 Chen Y, Liu Q, Guo D, et al. Emerging coronaviruses; genome structure, replication, and pathogenesis. J Med Virol 2020.

3 Cai JH, Wang XS, YL G, et al. First case of 2019 novel coronavirus infection in children in Shanghai. Chin J Pediatr2020;58:86-7. (in Chinese).

4 National Children's Medical Center \& Children's Hospital of Fudan University's Guideline Formulating Team of Rapid Screening \& Clinical Practices for Suspected and Confirmed Cases of Pediatric Novel Coronavirus Infection or Pneumonia. Guideline of Rapid Screening \& Clinical Practices for Suspected and Confirmed Cases of Pediatric Novel Coronavirus Infection or Pneumonia. Chin J Evid Based Pediatr 2020;15:1-4. (in Chinese).

5 Guan W, Ni Z, Hu Y, et al. Clinical characteristics of 2019 novel coronavirus infection in China. medRxiv 2020.

6 Li Q, Guan X, Wu P, et al. Early transmission dynamics in Wuhan, China, of novel coronavirus-infected pneumonia. N Engl J Med 2020;382:1199-207.

7 Wang F-S, Zhang C. What to do next to control the 2019-nCoV epidemic? The Lancet 2020;395:391-3.

8 World Health organization. infection prevention and control during health care when novel coronavirus ( $\mathrm{nCoV}$ ) infection is suspected. Available: https://www.who.int/publications-detail/infectionprevention-and-control-during-health-care-when-novel-coronavirus-( ncov)-infection-is-suspected-20200125. [Accessed 16 Mar 2020].

9 National Health Commission of People's Republic of China. Guidelines for transmission and prevention of novel coronaviruses. Available: https://www.nhc.gov.cn/xcs/kpzs/202001/9e73060017d7 44aeafff8834fc0389 f4.shtml [Accessed 27 Jan 2020].

10 Wei G, Zhang N, Yu H, et al. Clinical characteristics of 2019 nove coronavirus in China. medRxiv 2020.

11 Society of Pediatrics, Chinese Medical Association, Editorial Board, Chinese Journal of Pediatrics. [Recommendations for the diagnosis, prevention and control of the 2019 novel coronavirus infection in children (first interim edition)]. Zhonghua Er Ke Za Zhi 2020;58:E004.

12 Shen K, Yang Y, Wang T, et al. Diagnosis, treatment, and prevention of 2019 novel coronavirus infection in children: experts' consensus statement. World J Pediatr 2020;22. 